\title{
OCT ANGIOGRAPHY
}

Author: Sanja Sefic Kasumovic, MD, PhD et all. Publisher: JAYPEE, The Health Science Publisher, Ltd., New Delhi, London, Panama. 2018; A4 format, hard cover, 180 pages. ISBN: 978-93-5270-331-9.

\section{Izet Masic}

doi: 10.5455/aim.2018.26.219-220 ACTA INFORM MED. 2018 SEPT; 26(3): 219-220 Received: Jul 20, $2018 \bullet$ Accepted: Jul 30, 2018

\section{(c) 2018 Izet Masic}

This is an Open Access article distributed under the terms of the Creative Commons Attribution NonCommercial License (http://creativecommons.org/ licenses/by-nc/4.0//) which permits unrestricted noncommercial use, distribution, and reproduction in any medium, provided the original work is properly cited.
The book OCT Angiography is a handwriting which presents a good example of establishing a correlation with world literature in the field of new, sophisticated ophthalmology diagnostics. It represents a concise and sufficiently detailed monography on the new OCTA diagnostic method in ophthalmology. It is very important to note that the author of the book used their own material with her own descriptions and comments on every single image included, which gives the material a great value and worthiness. The book has 24 chapters: 1) Optical Coherence Tomography Angiography; 2) Choroidal Neovascularization; 3) Dry Age-related Macular Degeneration; 4) Neovascular/Exudative/Wet Age-related Macular Degeneration; 5) Central Serous Retinography; 6) Polipoidal Choroidal Vassculopathy, Idiopathic Polipoidal Choreidal Vasculopathy; 7) Diabetic Retinopathy; 8) Degenerative Myopia) 9) Retinal Artery Occlusion; 10) Retinal Vein Oclussion; 11) Sybvnchysis Scientillans; 12) Posterior Vitreous Detachment; 13) Epiretinal Membrane, Cellophane Maculopathy and Macular Pucker; 14) Vitreomacular Traction Syndrome; 15) Macular Pseudohole; 16) Lamellar Macular Hole; 18) Full Thickness Macular Hole, Stage 4; 19) Toxoplasmic Chorioretinitis; 20) Albinism; 21) X-linked Juvenile Retinoschisis; 22) Radiation Retinopathy; 23) Glaucoma; 24) Optic Neuritis and Papilledema. Auhors of these chapers are: Aida Kasumovic, MD; Emir Cabric, MD, PhD; Maja Zivkovic, MD, PhD; Lejla Muhamedagic, MD, MSc; Orhan Lepara, MD, $\mathrm{PhD}$; Nesina Avdagic, MD; PhD and

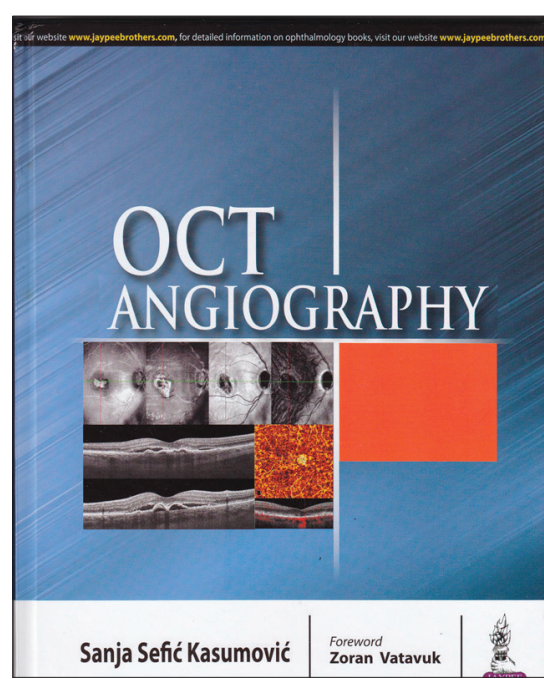

Tatjana Jeftic, MD. Authors are physicians and teachers from universities in Sarajevo and Tuzla (Bosnia and Herzegovina) and Nis (Serbia).

Some of pathological cases are presented with ten to fifteen individual images and figures, aiming to help the reader properly read and analyse the patient from beginning to the end. Also, images are organized so the reader can easily compare and understand correlation between them. Documented findings give the physician a possibility of careful comparison with earlier findings of the classic OCT. The author of this work has very skillfully introduced a reader into anatomy, physiology and pathology of the most common diseases of the posterior ocular segment. The book gives also a very good overview of optic nerve pathology and OCTA findings. MonographyOCT Angiography completes the requirements of educational literature from the domain of diagnostics the posterior eyesegment and can serve ophthalmologists, resi- 
dents of ophthalmology as well as medical students.

The book OCT angiography represents a handy guide through the OCTA technique while illustrating and explaining various retinal diseases as well as pathology of the optic nerve. The reader is provided with a short description of epidemiology, etiology, clinical signs and symptoms, as well as diagnostic and treatment possibilities for every entity described. The book is full of illustrations, colored figures and maps of every single case from everyday clinical practice and it provides a detailed step-by-step explanation below the every figure, with the aim of better insight and logical understanding of the retinal pathology. It is easy-to-read material, intended for medical students, residents of ophthalmology and ophthalmology specialists who are willing to expand their knowledge and to flow into the new technique of retina vascularization imaging. Every single case is represented by multiple images, which are arranged one-by-one, aiding in easy pattern recognition, diagnosis and monitoring of patients. It is updated diagnostic guidance for glaucoma experts who are willing to find out the real semiology of glaucoma development.

Sarajevo, September, 05th 2018

Prof Izet Masic, MD, PhD

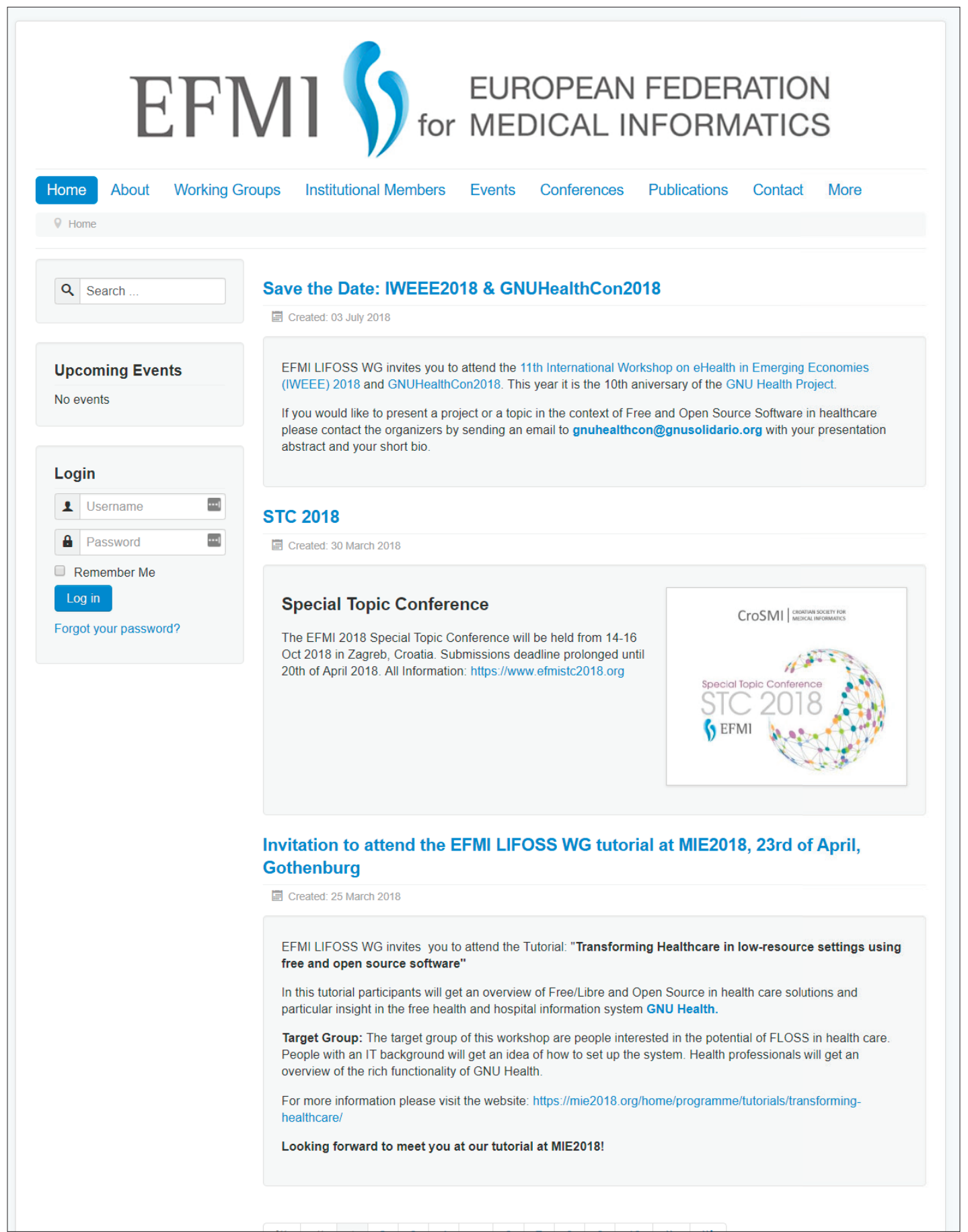

